\title{
On the Limit Behavior for Multi-Agent Dynamical Systems *
}

\author{
Ionela Prodan ${ }^{*, * *}$ Georges Bitsoris ${ }^{* * *}$ Sorin Olaru* \\ Cristina Stoica* Silviu-Iulian Niculescu** \\ * SUPELEC Systems Sciences (E3S) - Automatic Control Department, \\ France, ionela.prodan,sorin.olaru,cristina.stoica@supelec.fr. \\ ** CNRS-SUPELEC - Laboratory of Signal and Systems, France, \\ ionela.prodan,silviu.niculescu@lss.supelec.fr. \\ *** Control Systems Laboratory, Electrical Engineering Department, \\ University of Patras, Greece, bitsoris@ece.upatras.gr.
}

\begin{abstract}
This paper addresses the optimal control of multiple linear agents in the presence of a set of adversary constraints. This type of constraints makes the convergence of the agents' dynamics towards the "natural" equilibrium position, impossible to fulfill. Therefore, this default equilibrium point has to be replaced by a set of equilibrium points or even accept the existence of limit cycles. Furthermore, the constraints introduced in the transitory optimization problem are non convex. The present paper proposes a dual-mode control strategy which switches between an unconstrained optimum controller and a constrained control law whenever the adversary constraints are activated. The proposed method builds on invariance concepts and proves to be related to eigenstructure assignement problems. The technique exhibits effective performance and is validated here by an illustrative example.
\end{abstract}

Keywords: Multi-Agent Systems, positive invariance, non convex constraints, constrained MPC

\section{INTRODUCTION}

In many application involving the cooperative control of multiple agents [Grundel et al., 2007], collision avoidance represents a fundamental issue that needs to be integrated in the design strategy (see, for instance, [Richards and How, 2002]). This problem turns out to be a difficult one by the non-convexity of the associated constraints (see, for details [Stoican et al., 2011], at least from the computational point of view). These constraints are defined in the context of an autonomous agent navigating by the obstacles and/or other moving entities. Whenever the agent employs a continuous cycle of sensing and acting, a collision-free control law for the agent must be computed in each cycle based on the local observation of the environment.

The problem of collision avoidance has been extensively studied [Grundel and Pardalos, 2004]. Various control methods are related with the potential field approach [Tanner et al., 2007], or approaches based on graph theory [Lafferriere et al., 2005]. Alternative methods are based on Mixed Integer Programming (MIP)(see the comprehensive monography [Jünger et al., 2009]), which has the ability to include non convex constraints and discrete decisions in the optimization problem. However, despite its modeling capabilities and the availability of good solvers, MIP has serious numerical drawbacks. As stated in [Garey and Johnson, 1979], mixed-integer techniques are NP-hard, i.e. the computational complexity increases exponentially with the number of binary variables used in the problem

\footnotetext{
* The research of Ionela Prodan is financially supported by the EADS Corporate Foundation (091-AO09-1006).
}

formulation. A method for reducing the number of binary variables together with an application in the obstacles avoidance problem is detailed in [Stoican et al., 2011].

The goal of the present paper is twofold. In a first stage we perform a detailed analysis of the limit behavior for an agent with linear dynamics in the presence of adversary constraints. More precisely, we need to define the fixed points and the invariance properties for the agent state trajectory while avoiding a convex region containing the origin in its strict interior. This region can, in fact, represent an obstacle (static constraints) or another agent (dynamic constraints, leading to a parametrization of the set of constraints with respect to the current state).

In a second stage, our interest is to ensure the stability over the feasible region of the state space using a dualmode strategy. The principles can be found in the finite horizon formulation of Model Predictive Control (MPC) technique (see, for instance, [Mayne et al., 2000], for basic notions in MPC) including avoidance constraints for an agent. The first remark is that the presence of a restricted region constraint leads to the infeasibility of the control law around the origin (which is an equilibrium point for the autonomous system). This is an unusual formulation for the classical MPC design. To the best of the authors knowledge, all the studies on constrained MPC rely on the assumption that the origin is in the relative interior of the feasible region (see, for example, [Mayne et al., 2000], [Seron et al., 2002], [Bemporad et al., 2002]) or on the frontier of the feasible region [Pannocchia et al., 2003]. In the present paper we show that necessary and sufficient conditions can be formulated in order to 
guarantee the existence of a stable equilibrium point having the entire feasible region as a basin of attraction. The employed methods are specific for Piecewise Affine (PWA) systems analysis, with a geometric insight on the invariance properties of polytopic regions in the state space.

There are many applications of the present work which are of particular interest, namely those where static or dynamic constraints must be respected. Examples include coordinated ocean platform control for a mobile offshore base [Girard et al., 2001]. The homogeneous modules forming the offshore base must be able to perform long-term station keeping at sea, in the presence of waves, winds and currents. Therefore, the independent modules have to be controlled in order to be maintained aligned. This task can be easily accomplished if each module converges to different fixed points, which are suitably chosen. Furthermore, it is well known that the North Atlantic is one of the most inhospitable environments on the planet. Yet, it is here that Atlantic Norway's offshore oil and gas industry (see, [Grant and Shaw, 2001], [Bertino and Lisæter, 2008]) has been operating for years and with an impressive amount of success. In order to remain prosperous under such harsh conditions, the offshore industry relies on a variety of innovative technologies. An example is the ice-breaking cargo vessel and/or tanker which needs to break the ice around the platform. Therefore, the ice-breaking vessel has to maneuver as close as possible to the platform, while avoiding the collision with it (i.e. the vessel has to converge to a limit cycle).

The rest of the paper is organized as follows. In Section 2 the constrained predictive control problem is formulated. Section 3 presents the local constrained control problem based on invariance concepts, while the designed problem is developed in Section 4. Discussions based on the simulation results are presented in Section 5 and the conclusions are drawn in Section 6.

The following notations will be used throughout the paper. The spectrum of a matrix $M$ is the set of the eigenvalues of $M$, denoted by $\Lambda(M)=\left\{\lambda_{i}: i \in \mathbb{N}\right\}$. A point $x_{e}$ is a fixed point of a function $f$ if and only if $f\left(x_{e}\right)=x_{e}$ (i.e. a point identical to its own image). The boundary of a set $S$, denoted by $\partial S$ is the set of points which can be approached both from $S$ and from the outside of $S$.

\section{PRELIMINARIES}

Consider the behavior of an agent described by a discrete time linear time-invariant system:

$$
x_{t+1}=A x_{t}+B u_{t},
$$

where $x_{t} \in \mathbb{R}^{n}$ is the state, $u_{t} \in \mathbb{R}^{m}$ is the input signal and $A, B$ are state matrices of appropriate dimensions. It is assumed that the pair $(A, B)$ is stabilizable.

The goal is to control the agent such that its state is transferred as close as possible to the origin while its trajectory avoids a polyhedral region ${ }^{1}$ defined by:

$$
S=\left\{x \in \mathbb{R}^{n}: h_{i}^{T} x<k_{i}, \quad i=1: n_{h}\right\},
$$

\footnotetext{
1 Such limitations arise often in control applications and we will discuss later in the paper the collision or obstacle avoidance problems for multi-agent systems which originate them.
}

with $\left(h_{i}, k_{i}\right) \in \mathbb{R}^{n} \times \mathbb{R}$ and $n_{h}$ being the number of halfspaces. This paper focuses on the case where $k_{i}>0$ for all $i=1: n_{h}$, meaning that the origin is contained in the strict interior of the polytopic region, i.e. $0 \in S$. Note that, the feasible region in which the agent can reside is the non convex region defined as the complement $\mathbb{R}^{n} \backslash S$.

The minimization of a given cost function (usually a quadratic function involving states and inputs) leads to the linear state-feedback control law characterizing the optimal unconstrained infinite horizon problem:

$$
u_{t}=K_{L Q} x_{t}
$$

with $K_{L Q}$ computed from the solution of the discrete algebraic Riccati equation. However, here we want to design a control action such that the agent trajectories evolve outside the interdicted region (2):

$$
x_{t} \notin S \text {. }
$$

This can be enforced by the construction of an optimal control sequence $\mathbf{u}=\left\{u_{t \mid t}, u_{t+1 \mid t}, \cdots, u_{t+N-1 \mid t}\right\}$ over a finite constrained receding horizon. This leads to a predictive control policy:

$$
\begin{aligned}
\mathbf{u}^{*}=\underset{\mathbf{u}}{\arg \min }\left(x_{t+N \mid t}^{T} P x_{t+N \mid t}\right. & +\sum_{i=1}^{N-1} x_{t+i \mid t}^{T} Q x_{t+i \mid t}+ \\
& \left.+\sum_{i=0}^{N-1} u_{t+i \mid t}^{T} R u_{t+i \mid t}\right)
\end{aligned}
$$

Here $Q=Q^{T} \succeq 0, R \succ 0$ are positive definite weighting matrices, $P=P^{T} \succeq 0$ defines the terminal cost and $N$ denotes the prediction horizon.

In this classical convex formulations (even including input/state restrictions), the stability is assured by explicitly requiring that the state enters into a terminal region (containing the origin) at the end of the prediction horizon [Mayne et al., 2000].

Such considerations do not apply to the case:

$$
\begin{aligned}
& \mathbf{u}^{*}=\underset{\mathbf{u}}{\arg \min }\left(x_{t+N \mid t}^{T} P x_{t+N \mid t}+\sum_{i=1}^{N-1} x_{t+i \mid t}^{T} Q x_{t+i \mid t}+\right. \\
& \left.+\sum_{i=0}^{N-1} u_{t+i \mid t}^{T} R u_{t+i \mid t}\right) \\
& \text { s.t.: }\left\{\begin{array}{l}
x_{t+i+1 \mid t}=A x_{t+i \mid t}+B u_{t+i \mid t} \\
x_{t+i \mid t} \in \mathbb{R}^{n} \backslash S, \quad i=1: N
\end{array}\right.
\end{aligned}
$$

as long as the equilibrium point is not "approachable". Moreover, the non convex nature of the feasible region (7) imposes the use of mixed integer techniques, which means that auxiliary binary variables are added to the problem.

In order to reduce the complexity of the problem (6)(7), we propose a dual-mode control law which switches between an unconstrained optimum controller and a local constraints-handling feedback when necessary ${ }^{2}$.

\footnotetext{
2 Ideally we would like to have a controller which resembles the LQ control as mush as possible; in practice this means we can operate the LQ control when "far away" from the interdicted region and switch to the constrained control when approaching this region.
} 
Besides satisfying the constrains, we additionally impose that the agent state approaches an equilibrium point and avoids cyclic or chaotic behavior ${ }^{3}$.

\section{LOCAL CONSTRAINED CONTROL}

In this section, we first establish conditions for an affine state-feedback control law to both render a half-space positively invariant and to assign an equilibrium state lying on its boundary. Then, these conditions are used for the derivation of control law that transfer system's state as close as possible to the origin, all by avoiding the interdicted region.

Consider now an affine control law of the form:

$$
u_{t}=K\left(x_{t}-x_{e}\right)+u_{e},
$$

with $x_{e} \in \mathbb{R}^{n}$ the desired equilibrium state and $K \in$ $\mathbb{R}^{m \times n}$ the gain matrix. The resulting closed-loop system is described by the state equation

$$
x_{t+1}=A x_{t}+B K\left(x_{t}-x_{e}\right)+B u_{e},
$$

and $x_{t}-x_{e}$ defines its transient behavior.

A state $x_{e}$ is an equilibrium state for the closed-loop system (1) if:

$$
x_{e}=A x_{e}+B u_{e} .
$$

Therefore, only the states $x_{e}$ belonging to the preimage through the linear map $(I-A)$, of the linear subspace, spanned by the columns of matrix $B$, can represent equilibrium states.

Remark 1. The above conditions are independent of $K$. Consequently, the states that can be equilibria are defined by the dynamics of the unforced dynamics and are completely specified by $u_{e}$.

\subsection{Invariance guarantee}

With these elements, we can concentrate on one of the key issues for the control design: the controlled invariance with respect to an affine control law (8) and subsequently the closed-loop stability. For solving this problem, the following lemma describing algebraic invariance conditions will be used. Note that this result is a special case of a more general result established in [Bitsoris and Truffet, 2011].

Lemma 2. The half-space defined by the inequality:

$$
v^{T} x \leq \gamma,
$$

is a positively invariant set of the affine system

$$
x_{t+1}=M x_{t}+c,
$$

if and only if there exists a positive real number $g$ such that:

and

$$
v^{T} M=g v^{T}
$$

$$
g \gamma+v^{T} c \leq \gamma
$$

Proof: Suppose $x_{t}$ verifies relation (11):

$$
v^{T} x_{t} \leq \gamma
$$

\footnotetext{
3 In the general case the periodic solutions can be considered as optimal candidates for the limit behavior. In the present paper, the control objective is to avoid limit cycles and concentrate on the convergence to a fixed point.
}

We want to prove that $x_{t+1}$ verifies the same relation. By explicitly replacing:

$$
v^{T} x_{t+1}=v^{T}\left(M x_{t}+c\right)=g v^{T} x_{t}+v^{T} c
$$

and taking into account the hypothesis (15) and $\mathrm{g}$ is a positive number it follows that

$$
v^{T} x_{t+1} \leq g \gamma+v^{T} c \leq \gamma
$$

which is verified by (14).

According to Lemma 2, similar conditions will guarantee the positive invariance of the opposite half-space, defined by the inequality $v^{T} x \geq \gamma$. Thus, we establish the following result.

Theorem 3. If $x_{e}$ is an equilibrium state of the closedloop system (1) lying on the hyperplane $v^{T} x=\gamma$, then a necessary and sufficient condition for the hyperplane to partition the state space into two positively invariant halfspaces is that $v^{T}$ is a left eigenvector of the closed-loop matrix $A+B K$ associated with a positive eigenvalue $\lambda \leq 1$.

Proof: We apply the result from Lemma 2 to system (9) which can be written as:

$$
x_{t+1}=(A+B K) x_{t}+B\left(u_{e}-K x_{e}\right) .
$$

Then, the conditions (13)-(14) become:

$$
\begin{aligned}
& v^{T}(A+B K)=\lambda v^{T}, \\
& \lambda \gamma+v^{T} B\left(u_{e}-K x_{e}\right) \leq \gamma .
\end{aligned}
$$

Condition (20) is always satisfied if $x_{e}$ is an equilibrium state of the closed-loop system (18) lying on the hyperplane $v^{T} x=\gamma$. Indeed, in this case $x_{e}$ satisfies relation (10) and $v^{T} x_{e}=\gamma$. Then, condition (20) becomes:

$$
\lambda \gamma+v^{T} x_{e}-v^{T}(A+B K) x_{e} \leq \gamma,
$$

or

$$
\lambda \gamma+\gamma-\lambda v^{T} x_{e} \leq \gamma
$$

Finally, we have that $v^{T} x_{e} \geq \gamma$ which is verified.

It is clear that if conditions (19)-(20) are satisfied, then this is also true for

$$
\begin{aligned}
& -v^{T}(A+B K)=\lambda\left(-v^{T}\right), \\
& \lambda(-\gamma)-v^{T} B\left(u_{e}-K x_{e}\right) \leq-\gamma
\end{aligned}
$$

which means that the opposite half-space is positively invariant.

As seen above, under mild assumptions, the eigenvector of a closed-loop matrix can partition the space into two complementary and invariant half-spaces. Here we are interested in the converse problem: Given a hyperplane, does it exist a certain structural constraint on the gain matrix which makes the resulting closed-loop matrix to have the hyperplane as an eigenvector? If not, which is the closest approximation possible (in the sense of the infinity norm)? The approach proposed here is related to the eigenstructure assignment.

Indeed, for the dynamics described by (1) and a given vector $v \in \mathbb{R}^{n}$, under controllability assumptions, there exists a scalar $\lambda \in \mathbb{R}$ and a matrix $K \in \mathbb{R}^{m \times n}$ such that the pair $\left(\lambda, v^{T}\right)$ is an eigenvalue/left eigenvector of matrix $(A+B K)$ and $K$ verifies the linear constraint

$$
v^{T} B \cdot K=w^{T},
$$

with $w \in \mathbb{R}^{n}$ and $w^{T} \triangleq v^{T}(\lambda I-A)$. Furthermore, we obtain that $v^{T}$ depends on the values of $\lambda$ and $w^{T}$ : 


$$
v^{T}=w^{T}(\lambda I-A)^{-1} .
$$

An optimization problem can be formulated in order to find an eigenvalue $\lambda>0$ and a parameter $w^{T}$ (and implicitly a condition over the gain matrix $K$ ) such that the associated left eigenvector is equal with a given hyperplane $v$ :

$$
\begin{aligned}
& \min _{\delta, \lambda, w} \delta \\
\text { s.t.: } \quad-\delta & \leq v^{T}(A-\lambda I)+w^{T} \leq \delta \\
& \delta \geq 0 \\
0 & <\lambda<1
\end{aligned}
$$

In the case when the optimal solution is $\delta^{*}=0$, the vector $v$ can be used for the separation of invariant half-spaces (as detailed in Lemma 2 and Theorem 3) with respect to the closed-loop dynamics. Moreover, the conditions imposed on the associated eigenvalue assure the contractiveness of the respective eigenvector.

The optimal argument of the LP problem (27) $w^{*} \in \mathbb{R}^{n}$, will be instrumental in the control design problem through a linear constraint upon the fixed gain matrix:

$$
K^{T} z=w,
$$

with $z \in \mathbb{R}^{m}$. The equation (28) is obtained from (25) by considering $v^{T} B=z^{T}$ under full-column rank hypothesis, concerning the matrix $\mathrm{B}$.

\subsection{Affine parametrization of the feedback policies}

As it can be seen from the left eigenstructure assignment described above, the main difficulty for proving the stability in the neighborhood of $x_{e}$ is imposed by the structural constraint on the gain matrix inherited from the invariance desideratum. This imposes a reformulation of the local control problem in order to identify the design parameters.

In the following, we will derive an affine parametrization of the feedback policies such that a fixed gain matrix $K$ can be used for feedback, all by respecting the constraint (28). Note that $z \in \mathbb{R}^{m}$ can be decomposed into two elements $z=\left[\begin{array}{ll}\hat{z} & \tilde{z}\end{array}\right]$ such that the element $\tilde{z} \in \mathbb{R}^{*}$ (a non-zero scalar) and $\hat{z} \in \mathbb{R}^{m-1}$. Decomposing, $K^{T} \in \mathbb{R}^{n \times m}$ similarly into $\hat{K}^{T} \in \mathbb{R}^{n \times m}$ and $\tilde{K}^{T} \in \mathbb{R}^{n \times(m-1)}$ we can express after simple algebraic manipulations $\hat{K}^{T}$ as a function of $w, \hat{z}$ and $\tilde{z}^{-1}$. Introducing this into the original equality (28) we obtain an affine relation where $K^{T}$ is in the left side and $w, \hat{z}, \tilde{z}$ and $\tilde{K}^{T}$ are in an affine relation in the right side.

With these developments one can obtain an affine description of matrix $K \in \mathbb{R}^{m \times n}$ using the independent parameters contained in $\tilde{K} \in \mathbb{R}^{(m-1) \times n}$ :

$$
K^{T}=\Gamma+\tilde{K}^{T} \cdot \Psi
$$

where $\Gamma \in \mathbb{R}^{n \times m}$ and $\Psi \in \mathbb{R}^{(m-1) \times n}$ are defined as follows:

$$
\Gamma=\left[\mathbf{0}_{n \times(m-1)} w \tilde{z}^{-1}\right], \Psi=\left[\mathbf{I}_{(m-1)}-\hat{z} \tilde{z}^{-1}\right] .
$$

Remark 4. Note that for $m=1$ in (29), the gain matrix is directly given by $\Gamma=w z^{-1}$ since for this particular case the subspace defining $\tilde{K}$ is null.

Illustrative example: We propose here an illustrative example of the reasoning leading to equations (29)-(30) and the subsequent values of the matrices involved. In this sense, let use consider a matrix $K \in \mathbb{R}^{2 \times 2}$ which respects the constraint (28). Then, we can write:

$$
\left[\begin{array}{ll}
k_{11} & k_{12} \\
k_{21} & k_{22}
\end{array}\right]\left[\begin{array}{l}
z_{1} \\
z_{2}
\end{array}\right]=\left[\begin{array}{l}
w_{1} \\
w_{2}
\end{array}\right]
$$

which is equivalent to:

$$
\underbrace{\left[\begin{array}{ll}
k_{11} & k_{12} \\
k_{21} & k_{22}
\end{array}\right]}_{K^{T} \in \mathbb{R}^{2 \times 2}}=\underbrace{\left[\begin{array}{cc}
0 & w_{1} z_{2}^{-1} \\
0 & w_{2} z_{2}^{-1}
\end{array}\right]}_{\Gamma \in \mathbb{R}^{2 \times 2}}+\underbrace{\left[\begin{array}{c}
k_{11} \\
k_{21}
\end{array}\right]}_{\tilde{K}^{T} \in \mathbb{R}^{2}} \underbrace{\left[\begin{array}{c}
1-z_{1} z_{2}^{-1} \\
1
\end{array}\right]}_{\Psi \in \mathbb{R}^{1 \times 2}} .
$$

where each of the vectors/matrices corresponds with the notation in (29)-(30).

A similar decomposition can be applied to another particular case, i.e. for $K \in \mathbb{R}^{3 \times 3}$ :

$$
\begin{aligned}
& \underbrace{\left[\begin{array}{lll}
k_{11} & k_{12} & k_{13} \\
k_{21} & k_{22} & k_{23} \\
k_{31} & k_{32} & k_{33}
\end{array}\right]}_{K^{T} \in \mathbb{R}^{3 \times 3}}=\underbrace{\left[\begin{array}{lll}
0 & 0 & w_{1} z_{3}^{-1} \\
0 & 0 & w_{2} z_{3}^{-1} \\
0 & 0 & w_{3} z_{3}^{-1}
\end{array}\right]}_{\Gamma \in \mathbb{R}^{3 \times 3}}+\underbrace{\left[\begin{array}{ll}
k_{11} & k_{12} \\
k_{21} & k_{22} \\
k_{31} & k_{32}
\end{array}\right]}_{\tilde{K}^{T} \in \mathbb{R}^{3 \times 2}} \\
& \underbrace{\left[\begin{array}{ccc}
1 & 0 & -z_{1} z_{3}^{-1} \\
0 & 1 & -z_{2} z_{3}^{-1}
\end{array}\right]}_{\Psi \in \mathbb{R}^{2 \times 3}} .
\end{aligned}
$$

\subsection{Local controller synthesis}

Using the previous parametrization, relation (29) can be introduced into the closed-loop matrix as follows:

$$
A+B K=A+B\left(\Gamma^{T}+\Psi^{T} \tilde{K}\right)=\left(A+B \Gamma^{T}\right)+B \Psi^{T} \tilde{K} .
$$

This leads to a reformulation of the original dynamics (1) into a formulation as described in the following:

$$
x_{t+1}=\underbrace{\left(A+B \Gamma^{T}\right)}_{\tilde{A}} x_{t}+\underbrace{B \Psi^{T}}_{\tilde{B}} \tilde{K} x_{t},
$$

where we denote $A+B \Gamma=\tilde{A}$ and $B \Psi^{T}=\tilde{B}$.

We can now state that the controllability (which implies the stabilizability of the overall system) of system (1) with the gain matrix subject to a condition of type (28) is equivalent with the controllability of system (35). In this case, the usual controllability tests (e.g. the gramian of controllability, controllability matrix) and design of gain matrix apply (e.g. pole placement, Linear Quadratic Regulator (LQR) or solving a Riccati equation).

In the present paper we choose to construct the controller $\tilde{K}$ from (35) with a LQ design using the solution of the discrete algebraic Riccati equation

$$
\tilde{A}^{T} P+P \tilde{A}-P \tilde{B} R^{-1} \tilde{B}^{T} P+Q=0 .
$$

Furthermore, assuming the system (35) is controllable and a suitable gain matrix $\tilde{K}=R^{-1} B^{T} P$ is known, it is simple to introduce it in (29) and to obtain the stabilizing $K$ for the original dynamics (1).

Figure 1 resumes the theoretical details discussed in this section by a graphical illustration. Therefore, $S \in \mathbb{R}^{2}$ is the interdicted region defined as in (2). All the equilibrium states lie on the hyperplane which trespass the origin (see, (10) and Remark 1). Solving the optimization problem (27), we find an eigenvector which approximates very 
well a hyperplane of the interdicted region. Moreover, the hyperplane partition the space into invariant half-spaces (see Lemma 2). As consequence, the equilibrium point $x_{e}$ that we are interested on, lies at the intersection of the aforementioned hyperplanes.

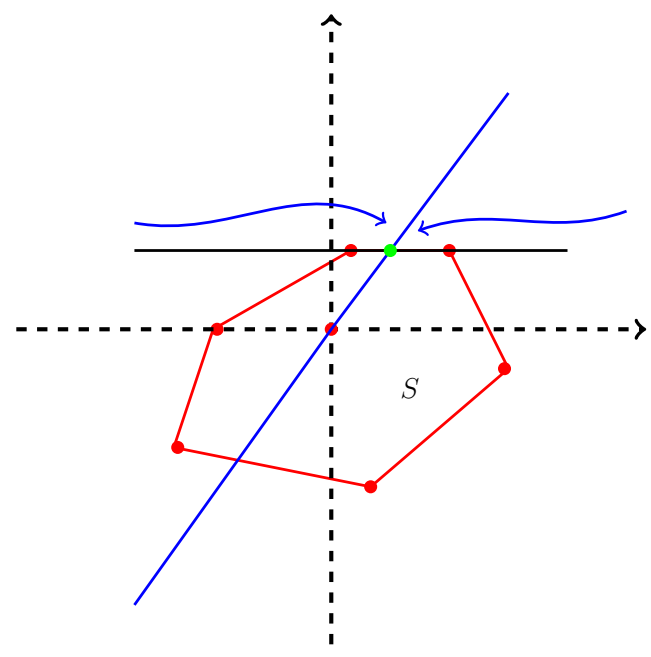

Fig. 1. The interdicted region and the equilibrium point, which lies on the boundary of the feasible region

\section{THE DESIGN PROBLEM}

With the results obtained in the previous section, a local linear control feedback gain is available such that $x_{e}$, a point on the frontier of $S$, is an attractor for the closed loop unconstraint trajectories. In the constrained case, the condition $x_{t} \notin S$ is assured only for a half-space, described by one of the supporting hyperplanes of $S$.

In the present section we will describe the procedure to ensure the stability of $x_{e}$ by the use of a receding horizon optimal control procedure. Its design principles are related to the dual-mode control:

- a generic optimization-based control integrating collision avoidance constraints;

- its equivalence with the unconstrained feedback law (8) over an invariant region containing $x_{e}$;

- guarantees of convergence in finite time towards this invariant region.

Consider the agent system (1) and the equilibrium point which verifies the relation $x_{e}=A x_{e}+B u_{e}$. The optimization problem to be solved is formulated as:

$$
\begin{aligned}
\mathbf{u}^{*}= & \underset{\mathbf{u}}{\arg \min }\left(\left(x_{t+N \mid t}-x_{e}\right)^{T} P\left(x_{t+N \mid t}-x_{e}\right)+\right. \\
& +\sum_{i=1}^{N-1}\left(x_{t+i \mid t}-x_{e}\right)^{T} Q\left(x_{t+i \mid t}-x_{e}\right)+ \\
& \left.+\sum_{i=0}^{N-1}\left(u_{t+i \mid t}^{T}-K x_{e}-u_{e}\right) R\left(u_{t+i \mid t}-K x_{e}-u_{e}\right)\right), \\
& \text { s.t.: }\left\{\begin{array}{l}
x_{t+i+1 \mid k}=A x_{t+i \mid t}+B u_{t+i \mid t}, \\
x_{t+i \mid t} \in \mathbb{R}^{n} \backslash S, \quad i=1: N,
\end{array}\right.
\end{aligned}
$$

with $\mathbf{u}=\left\{u_{t \mid t, \ldots, u_{t+N-1 \mid t}}\right\}$. The parameters $x_{e}, u_{e}$ and $K$, are determined in the previous section (see, (8)-(10), (28)-(35)). Applying the first component of the optimal formulation (37) and reiterating the optimization using the new state $x_{k}$, considered measurable, we dispose of a global control law with the following properties (formulated here without the formal proofs which can be derived without difficulties based on the classical results in [Chmielewski and Manousiouthakis, 1996] and [Mayne et al., 2000]):

- the optimization problem is recursively feasible (as consequence of the unbounded feasible domain);

- it is tractable (finite number of constraints);

- the matrices $P, Q, R$ can be tuned upon inverse optimality principles to ensure the the equivalence between the unconstrained optimum and the feedback control action (8), $u_{t}=K\left(x_{t}-x_{e}\right)+u_{e}$;

- there exists a prediction horizon such that the reachability analysis can be used to determine the minimal $N$ for which the predicted state trajectory

$$
v^{T} x_{t+N} \geq \gamma, \forall x_{t} \in\left(R^{n} \backslash S\right) .
$$

Furthermore, observe that the constraints introduced in (37) are non convex. This problem rises naturally from separation conditions (agents that have to avoid each others and/or obstacles, [Grundel and Pardalos, 2004], [Grundel et al., 2007]). As a solution to the collision avoidance problem, we use the well-known technique of Mixed-Integer Programming (MIP) for describing the feasible region. This implies that auxiliary binary variables are added to the problem. Since the non convex region is the exterior of a bounded convex set with finitely many support hyperplanes (a polytope), it is sufficient to add a binary variable for each hyperplane. However, by doing this, a significant number of binary variables is added in the problem formulation, thus leading to a sensitive numerical problem (the algorithms are branch and cut and in the worst-case scenarios are exponentially dependent of the number of binary variables). A method for reducing the computational time is detailed in [Stoican et al., 2011] with an application in an obstacles avoidance problem. This paper proposes a technique for making the time of computation P-hard in the number of LP/QP subproblems that have to be solved by means of a significant reduction in the number of binary variables.

Remark 5. Note that the initial optimization problem (37) is feasible since the constraints describe unbounded region. Here, for the simplicity of the presentation we let only constraints dealing with the state. Of course, a realistic approach requires constraints on the control action. We do not treat them here but we mention that their inclusion could make the problem more difficult to deal with, since the optimization problem may become infeasible for certain values of the input and states.

\section{SIMULATION RESULTS}

Consider an agent in two spatial dimensions with the dynamics described by:

$$
A=\left[\begin{array}{ll}
-0.78 & 0.33 \\
-0.85 & 1.08
\end{array}\right], \quad B=\left[\begin{array}{rr}
1 & 1 \\
-5 & 2
\end{array}\right]
$$

The components of the state are the position coordinates of the agent. Note that the pair $(A, B)$ is stabilizable. The state constraints as described in (2) are illustrated in Figure 2 by the red polytope. Solving the optimization problem (27), we obtained an affine parametrization of 
the gain matrix $K=\left[\begin{array}{rr}-0.17 & -0.09 \\ 0.74 & -0.38\end{array}\right]$ as in (29) with $\tilde{K}=\left[\begin{array}{ll}-0.17-0.09\end{array}\right], \Gamma=\left[\begin{array}{cc}0 & 0.86 \\ 0 & -0.31\end{array}\right]$ and $\Psi=\left[\begin{array}{ll}1 & 0.70\end{array}\right]$. This makes the closed-loop matrix to have a hyperplane of the interdicted region as an eigenvector. Furthermore, we obtained $u_{e}=\left[\begin{array}{ll}0.09 & 1.29\end{array}\right]$ and the equilibrium point $x_{e}=\left[\begin{array}{ll}0 & 10.1\end{array}\right]$, in Figure 2 is illustrated in green. The tunning parameters of the optimization problem (37) are: $P=\left[\begin{array}{rr}0.59 & -0.04 \\ -0.04 & 0.50\end{array}\right], \quad Q=\left[\begin{array}{ll}0.11 & 0.30 \\ 0.30 & 0.21\end{array}\right]$,

$R=\left[\begin{array}{rr}0.54 & -0.30 \\ -0.30 & 0.65\end{array}\right]$ and the prediction horizon $N=2$.

Finally, in Figure 2 we depict three different state trajectories converging to a unique equilibrium point when the predictive control law (37) is applied.

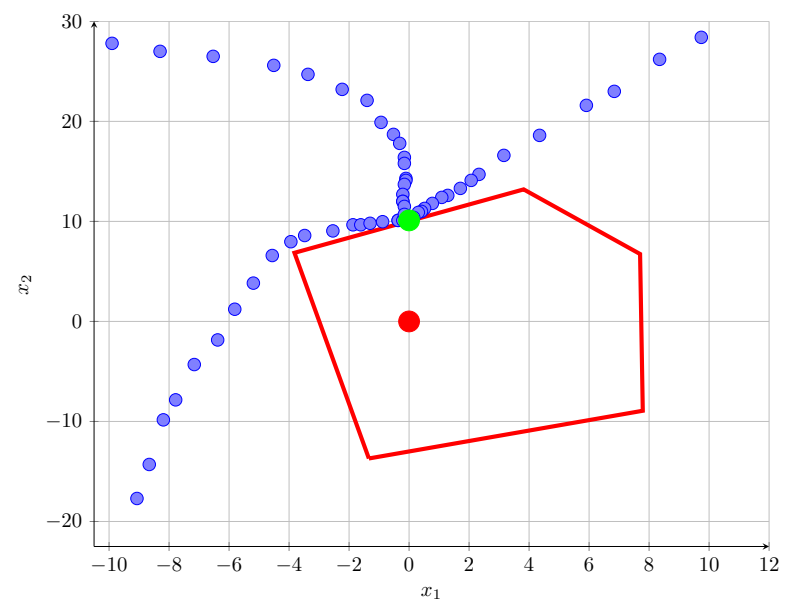

Fig. 2. The interdicted region and different agent state trajectories which converge to a fixed point.

\section{CONCLUSIONS}

A finite horizon predictive optimization problem formulation was proposed in order to describe the evolution of an agent in the presence of a set of adversary constraints. This type of constraints are particular, as they make the convergence of the agent trajectory to origin an infeasible task. We propose a dual-mode control law which switches between an unconstrained optimum controller and the predictive solution, when necessary. Simple algebraic conditions for the existence and uniqueness of a stable fixed point on the boundary of the feasible region represent the main result of this paper, completed with an optimization based control for the global attractivity. The analyzed cases are presented through some illustrative simulation results. Future work will focus on collision avoidance in the context of formations and the stabilization of multiple agents around a limit cycle.

\section{REFERENCES}

Bemporad A., Morari M., Dua V., and Pistikopoulos E.N. (2002): The explicit linear quadratic regulator for constrained systems. Automatica, vol. 38(1):3-20.
Bertino L. and Lisæter K.A. (2008): The topaz monitoring and prediction system for the Atlantic and Arctic oceans. Journal of Operational Oceanography, vol. $1(2): 15-18$.

Bitsoris G. and Truffet L. (2011): Positive invariance, monotonicity and comparison of nonlinear systems. Systems \& Control Letters, vol. 60(12):960-966.

Chmielewski D. and Manousiouthakis V. (1996): On constrained infinite-time linear quadratic optimal control. Systems \& Control Letters, vol. 29(3):121-129.

Garey M.R. and Johnson D.S. (1979): Computers and intractability. A guide to the theory of NP-completeness. A Series of Books in the Mathematical Sciences. WH Freeman and Company, San Francisco, California, 1979.

Girard A.R., Sousa J.B., and Hedrick J.K. (2001): Coordinated Control of Agent Formations in Uncertain, Dynamic Environments. In Proceedings of the European Control Conference, Porto, Portugal.

Grant C. and Shaw C. (2001): Operational oceanographic needs for the offshore oil and gas industry. GOOS data products and services bulletin, vol.1.

Grundel D. and Pardalos P.M. (2004): Theory and algorithms for cooperative systems, vol. 4. World Scientific Pub Co Inc.

Grundel D., Murphey R., and Pardalos P.M. (2007): Cooperative systems, Control and optimization, vol. 588. Springer Verlag.

Jünger M., Liebling T.M., Naddef D., Nemhauser G., and Pulleyblank W.R. (2009): 50 Years of Integer Programming 1958-2008: From the Early Years to the State-ofthe-Art. Springer Verlag.

Lafferriere G., Caughman J., and Williams A. (2005): Graph theoretic methods in the stability of vehicle formations. In Proceedings of the American Control Conference, vol. 4, pp. 3729-3734.

Mayne D.Q., Rawlings J.B., Rao C.V., and Scokaert P.O. (2000): Constrained model predictive control: Stability and optimality. Automatica, 36:789-814.

Pannocchia G., Wright S.J., and Rawlings J.B. (2003): Existence and computation of infinite horizon model predictive control with active steady-state input constraints. In IEEE Transactions on Automatic Control, vol. 48(6):1002-1006.

Richards A. and How J.P. (2002): Aircraft trajectory planning with collision avoidance using mixed integer linear programming. In Proceedings of the 21th American Control Conference, pp. 1936-1941, Anchorage, Alaska, USA.

Seron M.M., De Dona J.A., and Goodwin G.C. (2002): Global analytical model predictive control with input constraints. vol. 1, pp. 154-159.

Stoican F., Prodan I., and Olaru S. (2011): On the hyperplanes arrangements in mixed-integer techniques. In Proceedings of the 30th American Control Conference, pp. 1898-1903, San Francisco, California, USA.

Stoican F., Prodan I., and Olaru S. (2011): Enhancements on the hyperplane arrangements in mixed integer techniques. accepted to the 50th IEEE Conference on Decision and Control and European Control Conference, available upon request.

Tanner H.G., Jadbabaie A., and Pappas G.J. (2007): Flocking in fixed and switching networks. In IEEE Transactions on Automatic Control, vol. 52(5):863-868. 